\title{
Post-Covid-19 Beauty Industry
}

\author{
Soroush VALINIA *1,2 \\ ${ }^{1}$ Department of Research and Development, Tarah Teb Co., Tehran, Iran; \\ 2 Smart Beauty Co., Istanbul, Turkey \\ https://doi.org/10.15221/20.43
}

\begin{abstract}
The coronavirus pandemic has affected many industries, including the beauty industry. Beauty services and treatments and cosmetic procedures, which are considered elective and unnecessary, have been eliminated. Many believe that even if coronavirus diminishes, this pandemic might leave a lasting mark on how cosmetic care will be provided in the future. It is predicted that the beauty-related businesses will move into providing remote and online services pursuant to the use of novel technologies such as 3D scan and extended reality. We present an overview of the beauty industry possible changes after the coronavirus pandemic using the mentioned technologies and also introduce novel 3D-printed guides for cosmetic procedures.
\end{abstract}

Keywords: coronavirus, beauty industry, 3D scan, extended reality

\section{Introduction}

Many industries and businesses around the world are currently affected by the outbreak of the coronavirus, and the beauty industry is no exception. Most of the beauty salons and clinics in different countries have been closed, and unnecessary and elective surgeries have been stopped. Only patients who have an emergency or need follow-up visits are accepted. In this case, referral to the centers is done applying the social distancing, disinfecting surfaces and tools, and using Personal Protective Equipment (PPE).

Every year, many people travel overseas for medical purposes, including cosmetic surgery, to pay less or get better services, but now many of these trips have been stopped due to countries' entry bans.

Countries are at different stages of the coronavirus pandemic; some that were effective at initial containment, have seen a resurgence and are implementing additional measures to address it. Some others have seen the number of new cases begin to decline and are debating the right approach to reopening their economies.

Even as countries open up again, this pandemic leaves a lasting mark on how cosmetic care is provided around the world. Businesses need to consider what worked during the lockdowns, and use it to bring in a new era of the beauty industry. In this regard, it is predicted that many businesses will move into providing remote or online services, which requires the use of novel technologies. In this article, the possible changes of beauty industry after Coronavirus pandemic are discussed.

\section{Three-dimensional (3D) scan technology}

Many reliable sources have recommended the use of 3D imaging before cosmetic surgery, but only a few centers currently use this technology [1]. Perhaps this is a good opportunity to review the benefits of using 3D scanning and make the most of it. If affordable and small 3D scanners with user-friendly software and the ability to connect to mobile phones, TVs, and other home electrical appliances become available to most people, they can easily make their 3D models in related software (see figure 1). In this case, most of the needs related to beauty and health can be met online as follows in three categories, including cosmetic procedures, fashion, sport and fitness, without the need to leave home.

\footnotetext{
* CEO \& Founder of Smart Beauty Co.; soroush.valinia@smartbeautygroup.com; www.smartbeautygroup.com
} 


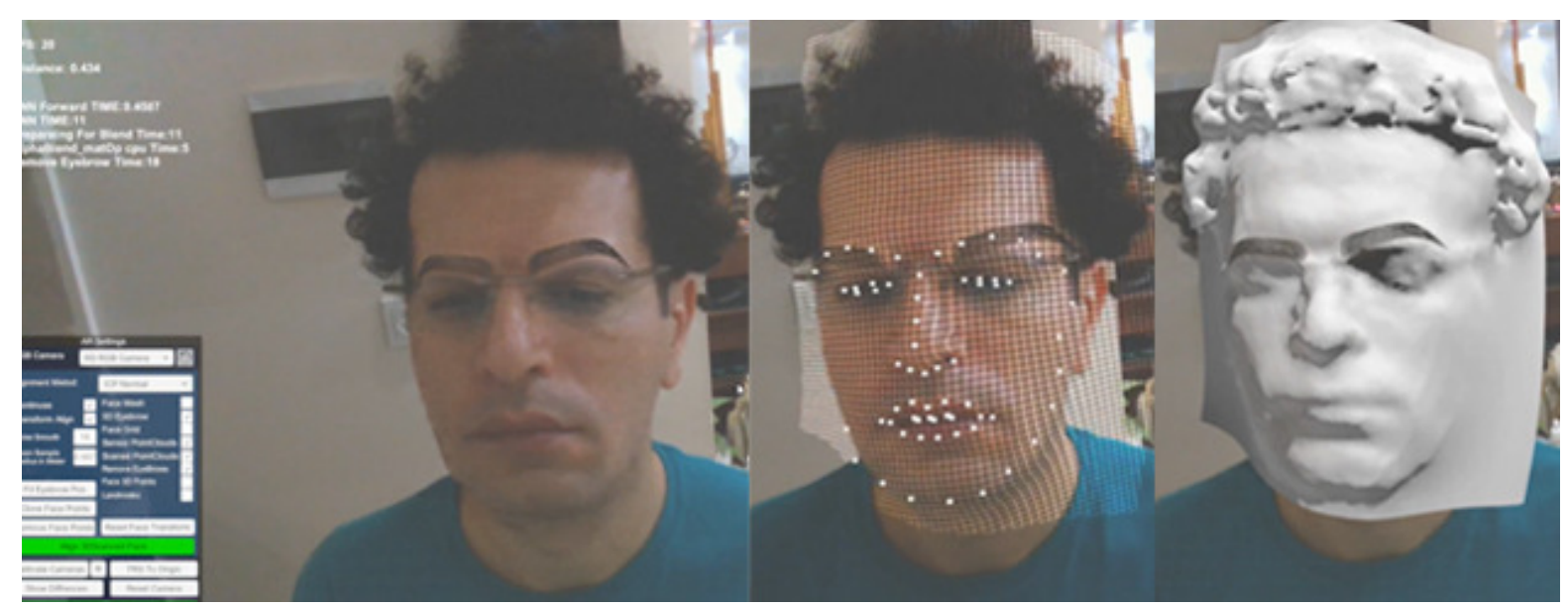

Fig. 1. Smart Beauty latest 3D scanner software.

\subsection{Cosmetic procedure}

With a 3D model, people can have an online consultation with plastic surgeons or beauticians. In this method, it is possible to apply changes and view the achievable results following the type of beauty procedure using related software; for example, a person can see the simulated result of rhinoplasty, breast surgery, facial filler injection, or even fat injection in different parts of their body or try different eyebrow microblading models on their face, and select the desired design according to their taste and cosmetologist's opinions. Also, by connecting the software to the virtual reality (VR) glasses, the person can see the simulated result in 3D from all angles close to reality. In this way, beauty counseling services will be provided without the need for in-person visits.

The online consultation, in this method, not only does not create any limitation but also has many benefits that help increase applicants' satisfaction. For example, by having a simulated 3D result, a set of guides and stencils can be 3D printed as an index to help doctors or beauticians apply changes accurately. In this method, the cosmetic procedure is completed when the result complies with printed guides. In this way, the probability of errors in beauty procedures is reduced, and the applicant will be more confident in achieving the desired simulated result.

In addition to 3D printed guides, 3D analyzes can have other benefits as well; for example, one of the new inventions helping increase the accuracy and quality of body augmentation and reduction cosmetic surgeries is real-time 3D scanning. One of the advantages of 3D simulation in this method is that by performing real-time scanning during the procedure and comparing the body shape with the simulated 3D model, doctors can detect injection points and the related amount of volume increase.

Another application of 3D scanners is for applicants' post-op visits. After surgery, doctors can analyze the 3D image of the operated body part and check the swelling, bruising, and shape daily without an inperson visit, and if necessary, apply preventive measures.

According to the mentioned applications, performing 3D scanning before the cosmetic procedure and using 3D printed guides will become necessary to reduce the likelihood of errors and applicants' dissatisfaction with final results. Also, using 3D scanners reduces the need to be present in doctors' waiting rooms and facilitates online consultation.

Smart Beauty Company has patented several inventions related to this subject, the detailed stages of which are provided hereafter (see figure 2) [2,3]. 

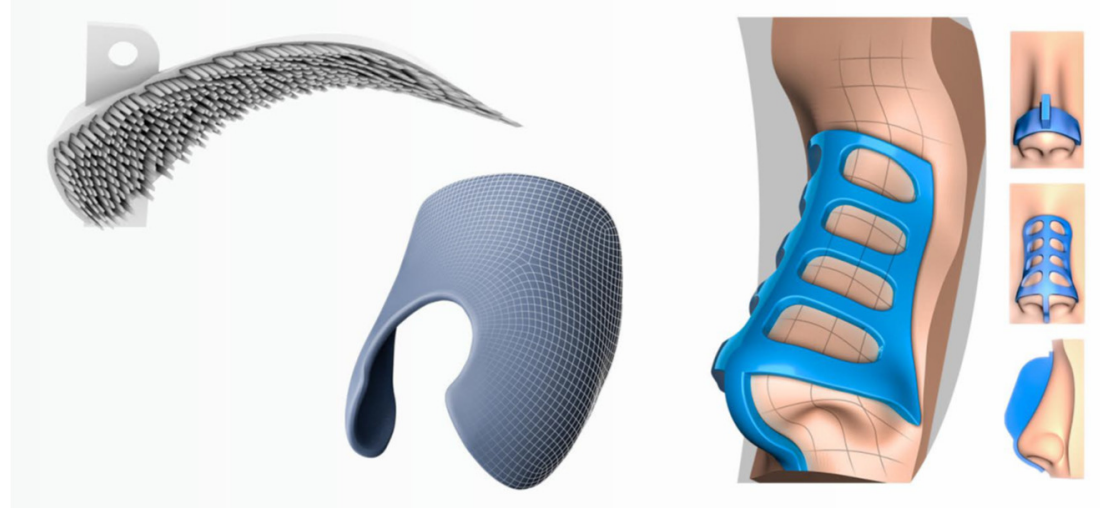

Fig. 2. Smart Beauty patented stencils and guides.

\subsubsection{Rhinoplasty using surgical guides}

Stages of rhinoplasty using surgical guides are: 1) 3D scanning and preparing the applicant's 3D model in the software; 2) Consultation with the surgeon, and apply changes based on the applicant's desires and surgeon's opinions on the nose 3D model; 3) Reaching to a mutual understanding between the applicant and the surgeon, and confirmation of the designed nose; 4) Production of the surgical guides with 3D printers based on the simulated 3D result; 5) Providing exact analyzes by comparing 3D models of the nose and the simulated result; 6) Performing rhinoplasty using Surgical guides and analyzes; 7) Examining the compliance of the surgical guide with the recently-operated nose (see figure 3).

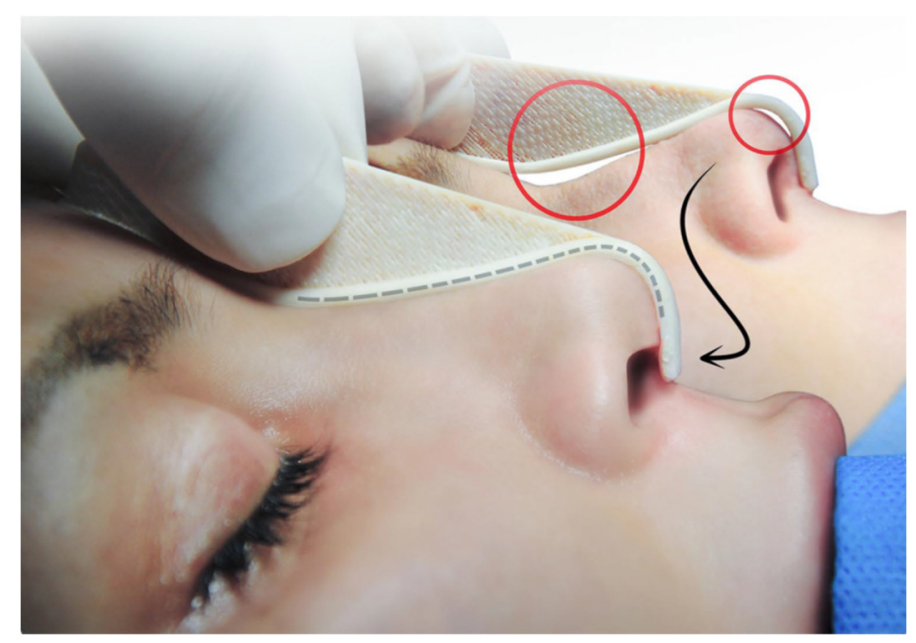

Fig. 3. Multi-dimensional compliance evaluation of rhinoplasty surgical guide with the nose shape.

\subsubsection{Face proportions control system for filler injection}

Stages of filler injection using face proportions control system are: 1) 3D scanning and preparing the applicant's 3D model in the software; 2) Consultation with the surgeon, and apply changes based on the applicant's desires and surgeon's opinions on the face 3D model; 3) Reaching to a mutual understanding between the applicant and the beauty professional, and confirmation of the designed face; 4) Providing exact analyzes by comparing 3D models of the face and the simulated result 5) Production of the injection guides with 3D printers based on the simulated 3D result. These guides are used during the injection to examine the accuracy of the changes made in different parts of the face (see figure 4). 


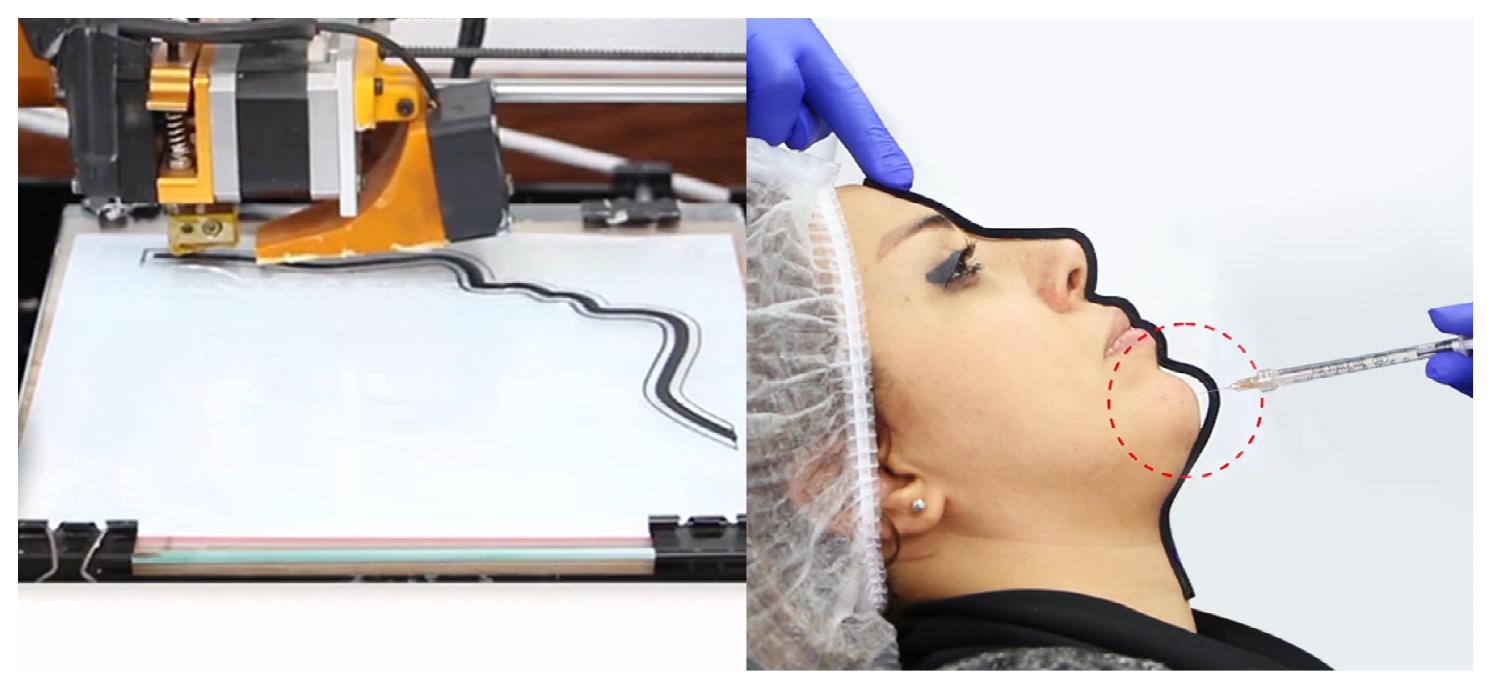

Fig. 4. 3D printed injection guide, and the use of the guide during filler injection.

\subsubsection{Eyebrow microblading using applicant customized stencil}

Stages of microblading using eyebrow stencils are: 1) 3D scanning and preparing the applicant's 3D model in the software; 2) Consultation with the beauty professional, and apply different eyebrow models based on the applicant's desires and professional opinions on the face 3D model; 3) Reaching to a mutual understanding between the applicant and beauty professional, and confirmation of designed eyebrow; 4) Producing an eyebrow stencil based on the simulated result; 5) Soaking the stencil with high durability ink and placing it on the face using artificial intelligence for proper positioning; 6) Tattooing the hair strokes marks which are based on the pre-designed eyebrow model (see figure 5).

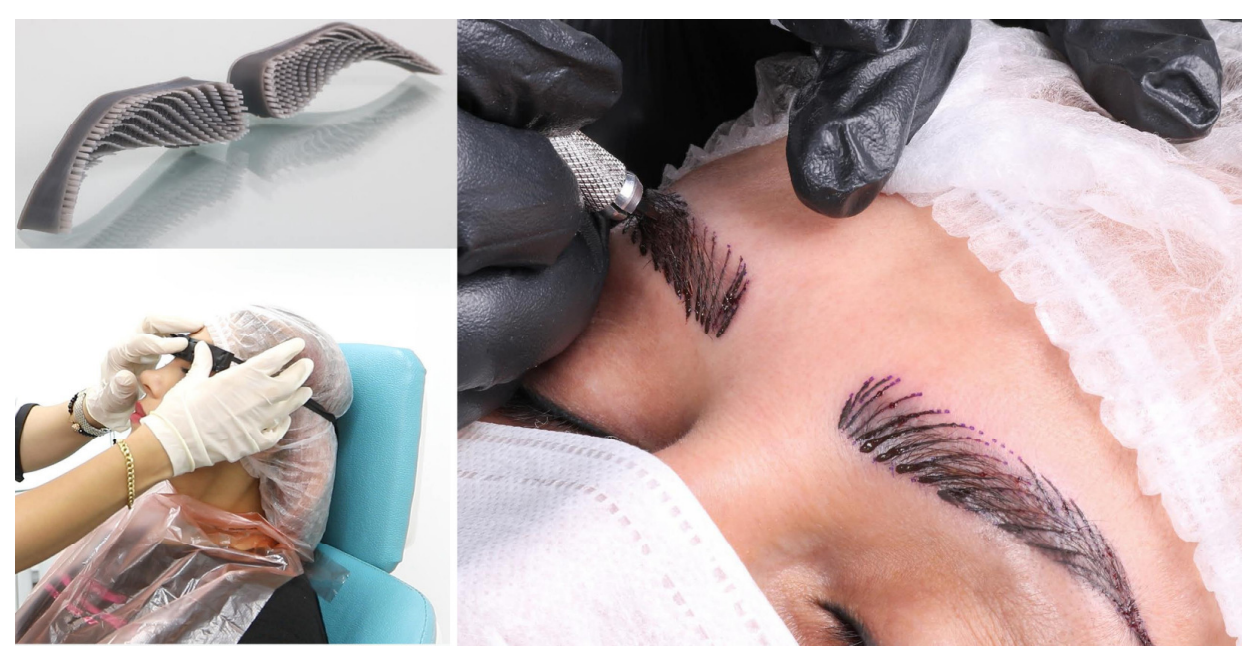

Fig. 5. Tattooing the hair strokes printed by eyebrow stencil.

\subsubsection{Reduction mammoplasty using printed stencils}

Stages of reduction mammoplasty using printed stencils are: 1) 3D scanning and preparing the applicant's 3D model in the software; 2) Consultation with the surgeon, and apply changes based on the applicant's desires and surgeon's opinions on the breast 3D model; 3) Agreement between the applicant and surgeon, and confirmation of designed breast; 4) Analyzing the volume of reduction in each breast, the distance between nipples, and the distance between each nipple to clavicle; 5) Producing stencils to specify breast incision lines based on the simulated result (see figure 6);6) Performing the surgery using stencils and exact analyzes. 

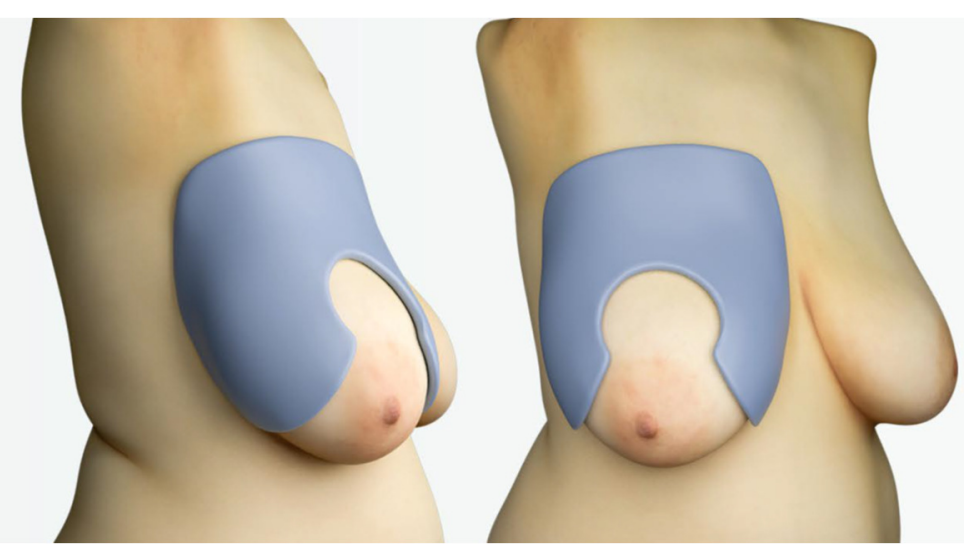

Fig. 6. Reduction mammoplasty stencil.

\subsection{Sport and fitness}

3D scanning technology can be a great tool for training and valued as a portable robotic trainer. A device comprising of a 3D scanner and processor can scan and evaluate body movements and warn if any activities are performed incorrectly. This feature can be an important factor in preventing sports injuries. This device can also analyze the relationship between a person's athletic performance and the music played and suggest the appropriate music associated with high efficiency. Furthermore, this device can be mobile or placed on an unmanned aerial vehicle such as a quadcopter and moves when the person changes position. The mentioned features reduce the need to go to health clubs or be trained by a coach [4].

3D scanning technology can also be useful for monitoring children's growth in Pediatric Clinical Examination. Doctors can analyze children's 3D body model at specific age intervals, and if necessary, prescribe appropriate medications or exercises without the need for an in-person examination. Another usage is that by analyzing real-time 3D scanning of the child's body during exercise, it will be possible to identify the child's sports talent more accurately.

3D scanning can be used in examining adults' body structural and anatomical changes, as well. By frequently analyzing persons' 3D body model and movement, doctors can predict the possibility as well as the probability of a hunchback or other spinal problems and give necessary treatment, including appropriate exercises, the use of medical belts or canes, etc. Also, doctors can evaluate body status and weight and suggest an appropriate diet online (see figure 7).

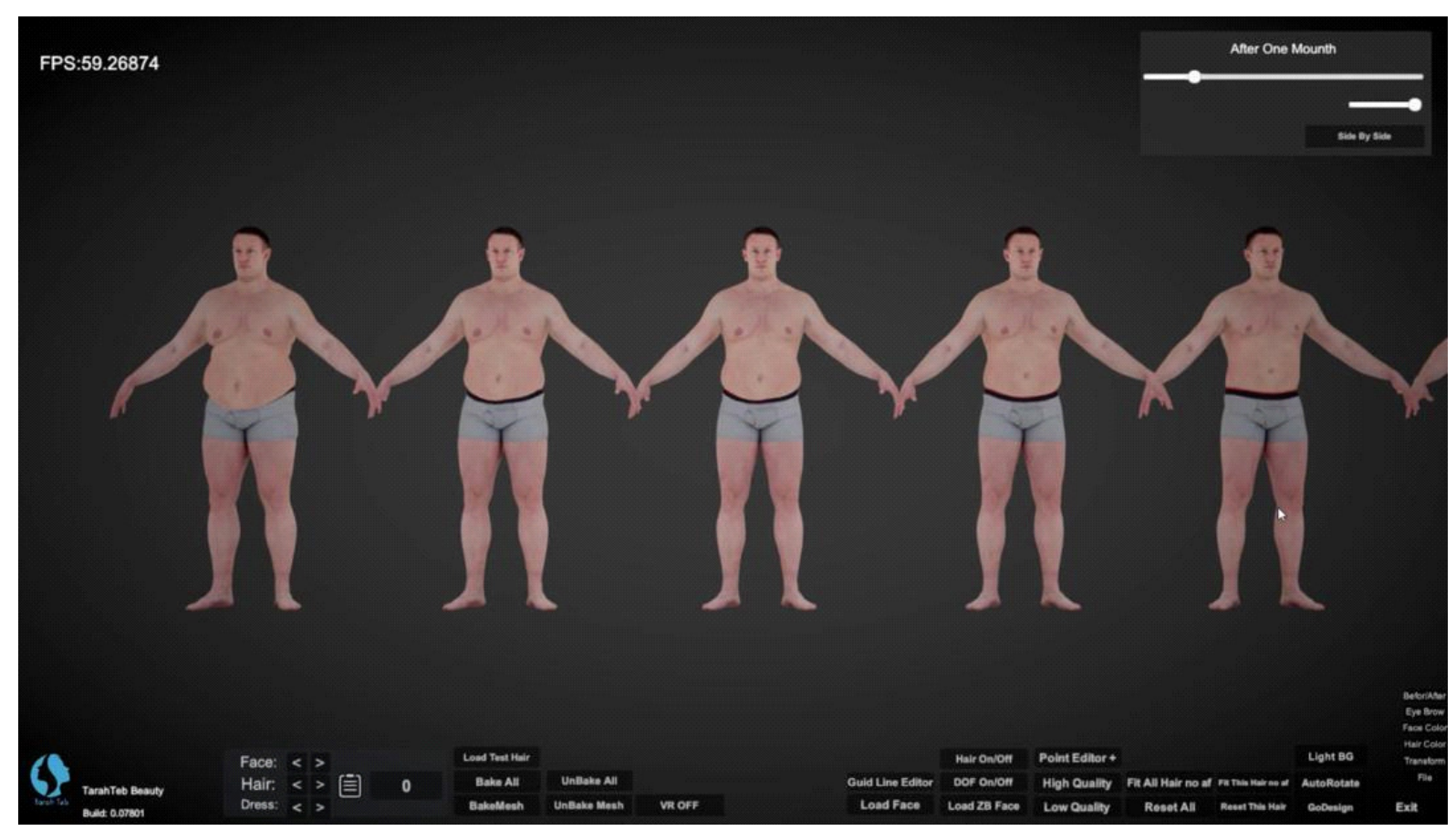

Fig. 7. Examining Edult's body anatomical changes. 


\subsection{Fashion}

By widespread distribution of these scanners in the market, reputable clothing brands can provide a suitable platform for customers to use various 3D home scanners to virtually try different clothes on their body and view it on the TV or using a VR glass. Since 3D models have accurate information about the size of the person's body, people can shop online and make sure that the size of the clothes ordered fits their bodies (see figure 8). This method can also be used in clothing design, through which people can select their desired texture, color, pattern, etc. and try the desired design on their 3D model and make online orders. Tailors can use these 3D designs to sew ordered clothes needless of in-person visits.

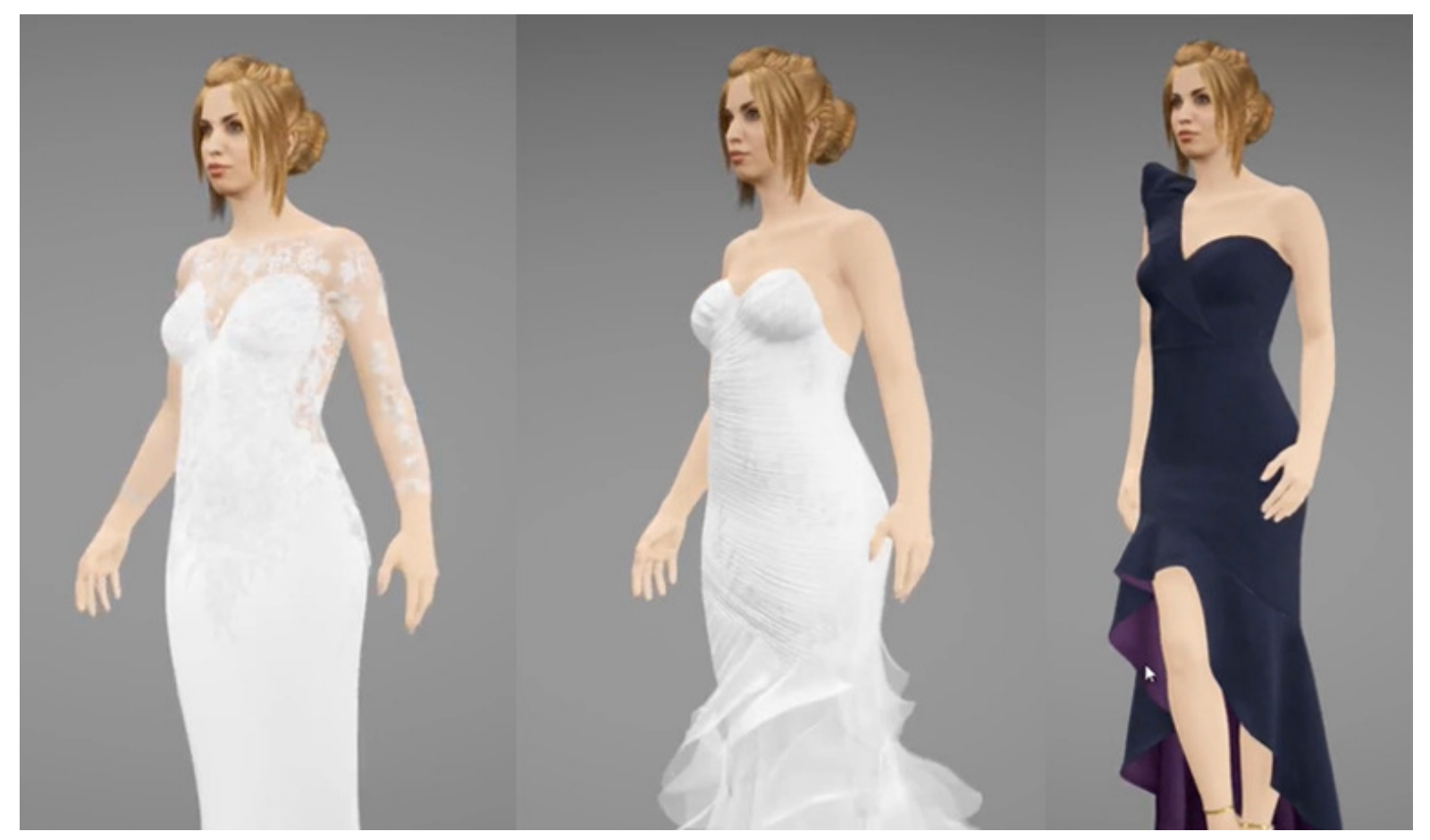

Fig. 8. Trying different clothes on a 3D body model.

\section{Extended reality technology}

After the virtual and augmented reality, mixed reality technology was introduced, through which it is possible to interact with digital objects added to the environment. These technologies, generally called extended reality, can be very helpful in different areas, such as education, design, and medicine, and change the experience of the world around us. It is predicted that the use of these technologies will also expand in cosmetic practices; for example, they can be used in learning cosmetic procedures virtually without the need to practice on actual patients. Also, using this technology, plastic surgeons or beauticians can have a virtual face to face consultation with applicants, and doctors can virtually examine their patients.

The use of these technologies in the training of various sports can also be very useful, and people can do the necessary exercises without the need to go to sports clubs and can do the exercise by him or herself. In this way, people can record their performance, and after replaying it, have a better understanding of their mistakes and correct their move and increase the performance. Many professional athletes use this method to practice and increase their skills, and that is expected to become more common for public use in the future.

In video games, people can use 3D scanning to build their avatars and play with each other. Also, with the help of extended reality technology, as well as a different and extraordinary experience, people would be in the game environment actively, thus preventing long-term sitting and develop musculoskeletal pain, due to decreased physical activity that lead to physical disorders. 


\section{References}

[1] C.P. Honrado and Jr. Wayne F. "Update in three-dimensional imaging in facial plastic surgery." Current opinion in otolaryngology \& head and neck surgery 12.4 (2004): 327-331.

[2] S.S. Valinia. "Rhinoplasty Cotrol Molds (transverse, Axial, Basal, Final, Final Splint)." U.S. Patent Application No. 15/179,982.

[3] S.S. Valinia, "Mark stencil and method for marking correct incision lines in reduction mammoplasty surgery." International Bureau of WIPO No. PCT/IB2018/060730.

[3] A.G. Bachrach et al. "Fitness and sports applications for an autonomous unmanned aerial vehicle." U.S. Patent Application No. 16/439,504. 